\title{
Engineering carbon materials with electricity
}

Article

Accepted Version

Creative Commons: Attribution-Noncommercial-No Derivative Works 4.0

Harris, P. J. F. (2017) Engineering carbon materials with electricity. Carbon, 122 (October). pp. 504-513. ISSN 00086223 doi: https://doi.org/10.1016/j.carbon.2017.06.084 Available at https://centaur.reading.ac.uk/71086/

It is advisable to refer to the publisher's version if you intend to cite from the work. See Guidance on citing.

Published version at: http://dx.doi.org/10.1016/j.carbon.2017.06.084

To link to this article DOI: http://dx.doi.org/10.1016/j.carbon.2017.06.084

Publisher: Elsevier

All outputs in CentAUR are protected by Intellectual Property Rights law, including copyright law. Copyright and IPR is retained by the creators or other copyright holders. Terms and conditions for use of this material are defined in the End User Agreement.

\section{www.reading.ac.uk/centaur}

\section{CentAUR}

Central Archive at the University of Reading

Reading's research outputs online 


\section{Accepted Manuscript}

Engineering carbon materials with electricity

Peter J.F. Harris

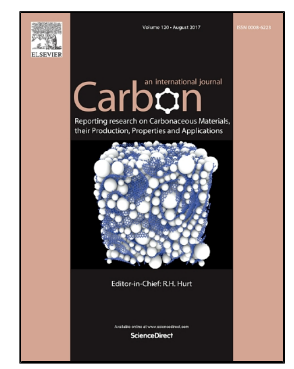

PII:

S0008-6223(17)30662-0

DOI:

10.1016/j.carbon.2017.06.084

Reference:

CARBON 12148

To appear in:

Carbon

Received Date:

26 May 2017

Revised Date:

23 June 2017

Accepted Date:

24 June 2017

Please cite this article as: Peter J.F. Harris, Engineering carbon materials with electricity, Carbon (2017), doi: 10.1016/j.carbon.2017.06.084

This is a PDF file of an unedited manuscript that has been accepted for publication. As a service to our customers we are providing this early version of the manuscript. The manuscript will undergo copyediting, typesetting, and review of the resulting proof before it is published in its final form. Please note that during the production process errors may be discovered which could affect the content, and all legal disclaimers that apply to the journal pertain. 


\section{Engineering carbon materials with electricity}

\section{Peter J.F. Harris*}

Electron Microscopy Laboratory, Department of Chemistry, J.J. Thomson Building, University of Reading, Whiteknights, Reading RG6 6AF, UK.

*Corresponding author. E-mail address: p.j.f.harris@reading.ac.uk 


\begin{abstract}
The passage of an electric current, or the application of an electrostatic charge, can be used to modify the structure of carbon materials in a variety of ways. The simplest example of these involves the use of high current densities to produce electrical breakdown, as a result of Joule heating or electromigration. This has been used by a number of groups to create gaps in carbon materials in order to fabricate devices such as transistors. More complex structural transformations have been observed when electric fields are applied to carbon nanomaterials. These involve the formation of carbon structures made up of single-layer or bilayer graphene with highly irregular morphologies, displaying many unusual features, including nanotubegraphene junctions. Although the nature of these transformations is disputed, they may be partly a result of electrostatic charging rather than the passage of an electric current. It has also been demonstrated that electric fields can be used to induce exfoliation of graphite, both on the nano scale and macroscopically. This article is an attempt to provide an overview of the different ways in which carbon materials can be engineered using electricity. In addition to pure carbons, work on doped and filled nanotubes is covered, and the possibility of using electric fields in "graphene origami" is discussed.
\end{abstract}




\section{Contents}

1. Introduction ...................................................................................................

2. Effects due to passage of a current ...................................................................

2.1 Carbon nanotubes ...................................................................................

2.2 Graphene ....................................................................................10

2.3 Disordered carbon ..................................................................................11

3. Effects apparently due to electrostatic charging ................................12

3.1 Structural transformation induced by electric field.......................12

3.2 The mechanism of structural transformation................................15

3.3 Electrostatic exfoliation........................................................17

3.4 Graphene origami .....................................................................................17

4. Experiments with doped and filled nanotubes ..........................................18

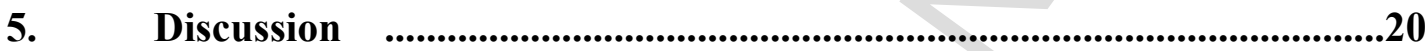

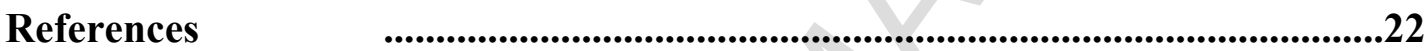

\section{Introduction}

Carbon and electricity have a shared history stretching back over 200 years [1]. The first electric light, the carbon arc lamp, was demonstrated by Humphry Davy some time around 1800, while incandescent electric lamps, utilising carbon filaments, were developed by Thomas Edison and Joseph Swan in the 1870s. The Hall-Héroult process, which uses carbon electrodes to smelt aluminium, was patented in 1889, and a short time later Acheson developed his electric furnace process for synthesizing graphite and silicon carbide. Today graphite is used as an anode in most batteries, and plays an essential role in power generation as a neutron moderator in nuclear reactors. Some of the most important developments in carbon science in recent decades have also involved electricity, including the bulk synthesis of $\mathrm{C}_{60}$ by arc discharge in 1990 [2] and the production of multiwalled carbon nanotubes in a similar way in the following year [3].

The aim of the present article is to review the ways in which carbon nanomaterials can be manipulated and modified using electricity. An attempt is made to distinguish between the 
effect of passing an electric current and the effect of the application of an electrostatic charge. The passage of a current can result in Joule heating, i.e. resistive heating, and electromigration, i.e. mass transport of carbon atoms due to the momentum transfer from conducting electrons to the graphene sheets. Both phenomena can contribute to structural breakdown when a sufficiently high current density is applied, as described in Section 2. In some cases, the experiments are carried out in the presence of oxygen, which can greatly promote breakdown. Electrical breakdown has been widely applied to carbon nanotubes, to create gaps in order to create devices such as transistors. Some very skilful studies of the mechanism of electrical breakdown of nanotubes, using in situ TEM, have been carried out, and these are described.

Another effect which has been observed when a current is passed through a carbon nanotube is superplasticity. It has been shown that applying a stress to a single-walled carbon nanotubes (SWCNT) through which a current is being passed can result in an elongation of up to $280 \%$. This remarkable phenomenon is discussed, together with experiments on the current-induced shrinkage of nanotubes. Outstanding work on the "plumbing" together of carbon nanotubes using an electric current is also described. The use of electrical breakdown to create gaps in graphene is then discussed, and an interesting study of the effect of passing an electric current on the structure of disordered carbons is described.

Section 3 describes effects which appear to be due, at least in part, to electrostatic charging. A number of studies over the past 8 years or so have demonstrated that dramatic structural transformations can be produced when carbon nanomaterials are exposed to an electric field at high temperatures. These transformations seem to differ from those produced by the passage of a current, and produce carbon materials made up of single-layer or bilayer graphene with highly irregular morphologies. The transformed carbons display many unusual features, including nanotube-graphene junctions and nanoparticles apparently encapsulated inside larger structures. Several studies of these transformations have now been reported, but the mechanism remains poorly understood. Some authors have suggested that the structural changes are a consequence of Joule heating, but it is argued here that electrostatic charging is the primary reason for the transformations. Theoretical work showing that the application of an electric field can reduce the van der Waals forces between adjacent graphene layers would seem to support this idea. The use of electric fields to induce the exfoliation of graphite is then discussed, and theoretical work on the ways in which fields could be used to control the 
folding and unfolding of graphene structures, in what has been called "graphene origami" is summarised.

Although this review is mainly focussed on the way in which electric fields can be used to modify pure carbons, work on doped carbon nanotubes, and on material encapsulated inside carbon nanotubes is also reviewed.

\section{Effects due to passage of a current}

\subsection{Carbon nanotubes}

It is well established that both carbon nanotubes and graphene exhibit exceptionally high breakdown current densities. Values as high as $4 \times 10^{9} \mathrm{~A} / \mathrm{cm}^{2}$ have been reported for SWCNTs [4], while a figure of $10^{8} \mathrm{~A} / \mathrm{cm}^{2}$ was found for $16 \mathrm{~nm}$ wide graphene nanoribbons [5]. These numbers are 2-3 orders of magnitude higher than that the values for typical metals, and are a result of the strength of the carbon-carbon bonds. When currents in excess of these values is applied, however, breakdown does occur, and can be used to modify the structures of both nanotubes and graphene in a reasonably controlled way.

Avouris and colleagues were among the first to use electrical breakdown to modify carbon nanotubes. They first applied the method to multi-walled nanotubes (MWCNTs) in 2001 [6, 7]. Breakdown was found to occur in a series of sharp current steps, as shown in Fig.1; this was attributed to the sequential destruction of individual nanotube shells. The failure was

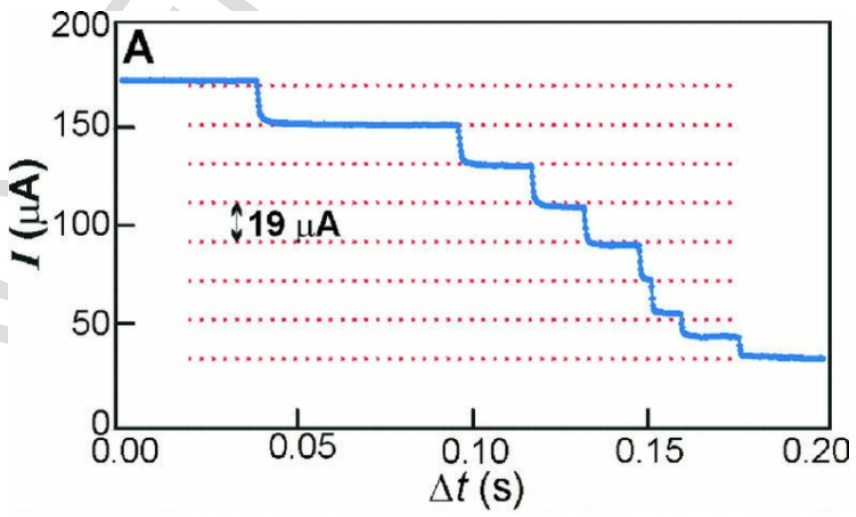

Fig. 1 Plot of current vs. time showing partial electrical breakdown of MWCNT at constant voltage, from the work of Avouris and colleagues [7]. 
found to occur much more readily in air than in vacuum, apparently as a result of oxidation. Thus, a $14 \mathrm{~nm}$ diameter MWNT experienced breakdown at a power of approximately 520 $\mu \mathrm{W}$ in vacuum and at $320 \mu \mathrm{W}$ in air. It was noted however that breakdown in vacuum occurred much more rapidly, once initiated. The same group also used current-induced electrical breakdown to selectively remove metallic tubes from "ropes" of single-walled carbon nanotubes SWCNTs [7]. By eliminating the metallic tubes, this group were able to fabricate nanoscale circuits based solely on semiconducting SWCNTs.

Since this early work, electrical breakdown of carbon nanotubes has been quite widely studied. A number of groups have used electrical breakdown to create gaps in nanotubes. For example, Dai and colleagues fabricated transistors by depositing organic materials in electrically-created gaps in SWCNTs [8]. The size of the cut was found to be controllable by varying the lengths of the SWCNTs, and gaps as small as $2 \mathrm{~nm}$ could be produced. In this work the cutting process was carried out under argon. Other groups have created gaps by passing a current in air $[9,10]$; in such cases the breakdown is presumably due to oxidation.

The mechanism of electrical breakdown has been studied by several groups using in situ TEM. One of the first such studies was described by Huang et al. in 2005 [11]. In this work, current was passed through MWCNTs while they were being imaged inside a TEM.

Breakdown behaviour was similar to that observed by Avouris et al. in that it occurred wallby-wall, sometimes from the outermost wall inward, and sometimes from the innermost wall outward. In other cases, breakdown of outer and inner walls in turn was observed. Figure 2 shows an example of the breakdown of an initially 6-wall nanotube from the outermost wall inward. The current passed through the tube was initially $240 \mu \mathrm{A}$, and sequential drops in current were observed as the walls were removed.

Further in situ studies of the breakdown of MWCNTs by Joule heating were described by Jin and colleagues in 2008 [12]. A particularly interesting feature of this work was that the edges of adjacent broken shells could bond together in a so-called lip-lip interaction in order to eliminate dangling bonds. This is illustrated in Fig. 3. The first stage, shown in Fig. 3 (a) involved the formation of open edges in the outer layers, indicated by blue arrows. The upper open edge then moved downwards, as shown in (b), and formed a lip-lip connection with the other edge, indicated by the red arrows in (c). The lip-lip connections were observed to 

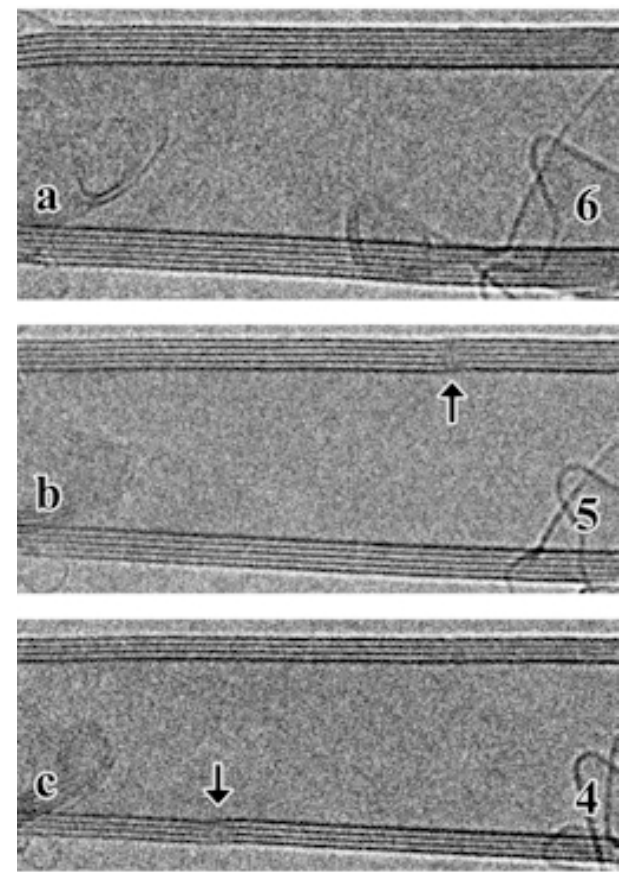

Fig. 2 (a) - (e) HRTEM images from the work of Huang et al. [11] showing breakdown of MWCNT as a result of passage of an electric

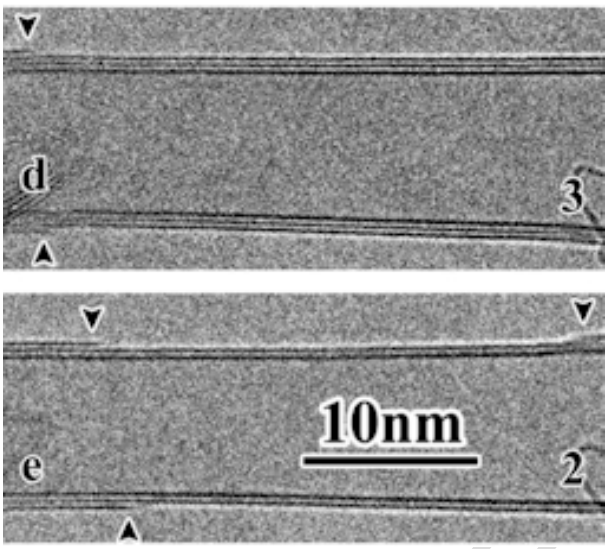
current. Breakdown occurs sequentially from the outer to the innermost wall. The arrows mark kinks, while the arrowheads denote the residue of the 4th and the 3rd walls after breakdown.

undergo structural fluctuations, especially when a higher biased voltage was applied, as can be seen in Fig. 3 (d). These were believed to be a result of a continuous reorganization of the bridging carbon bonds at high temperature. The authors suggested that such lip-lip interactions might stabilise the open-ended growth of MWCNTs in the arc discharge process.

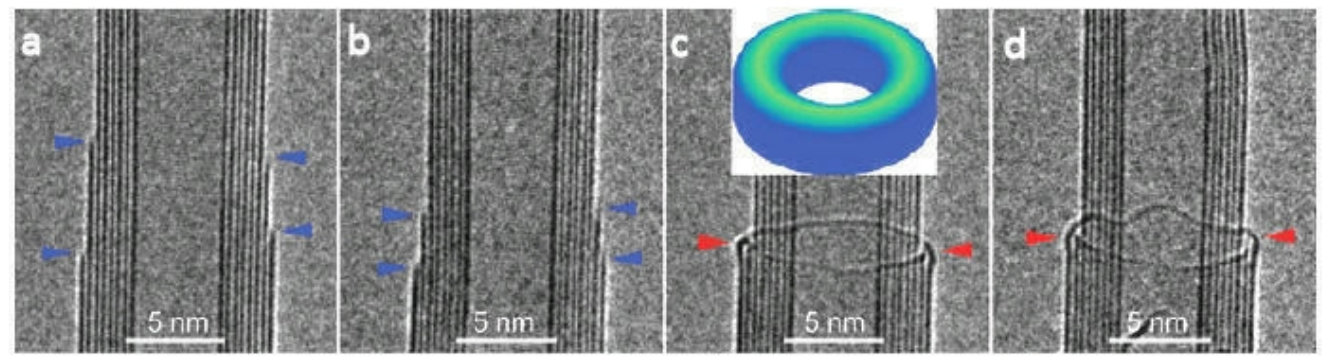

Fig. 3 (a) - (d) HRTEM images showing the formation of lip-lip bonding during breakdown of MWCNT as a result of Joule heating, from work by Jin and colleagues [12]. The inset in (c) is a schematic illustration of the lip-lip structure. 
In 2006, Huang and colleagues showed that when a current is passed through a carbon nanotube it can display extraordinary plasticity [13]. These workers used a piezo manipulator to apply a stress to a SWCNT, initially $24 \mathrm{~nm}$ long, through which a current was being passed. At tensile failure, the SWCNT had stretched to a length of $91 \mathrm{~nm}$ long, representing a tensile elongation of $280 \%$; its diameter was reduced 15 -fold, from 12 to $0.8 \mathrm{~nm}$, as can be seen in Fig. 4 . Tensile-pulling experiments at room temperature without any electric current showed that nanotubes typically failed at a tensile strain of less than $15 \%$. In their original paper [13], Huang et al. suggested that the mechanism of elongation involved the movement of 5-7 dislocation kinks through a relatively clean lattice. In subsequent work [14], Yakobson and colleagues suggested that sublimation of carbon atoms was also involved, occurring in the vicinity of 5-7 dislocation cores. Overall it seems that superplasticity in carbon nanotubes is still not fully understood, and it is not clear whether the process is promoted by the passage of a current or whether it is simply a consequence of the high temperature.

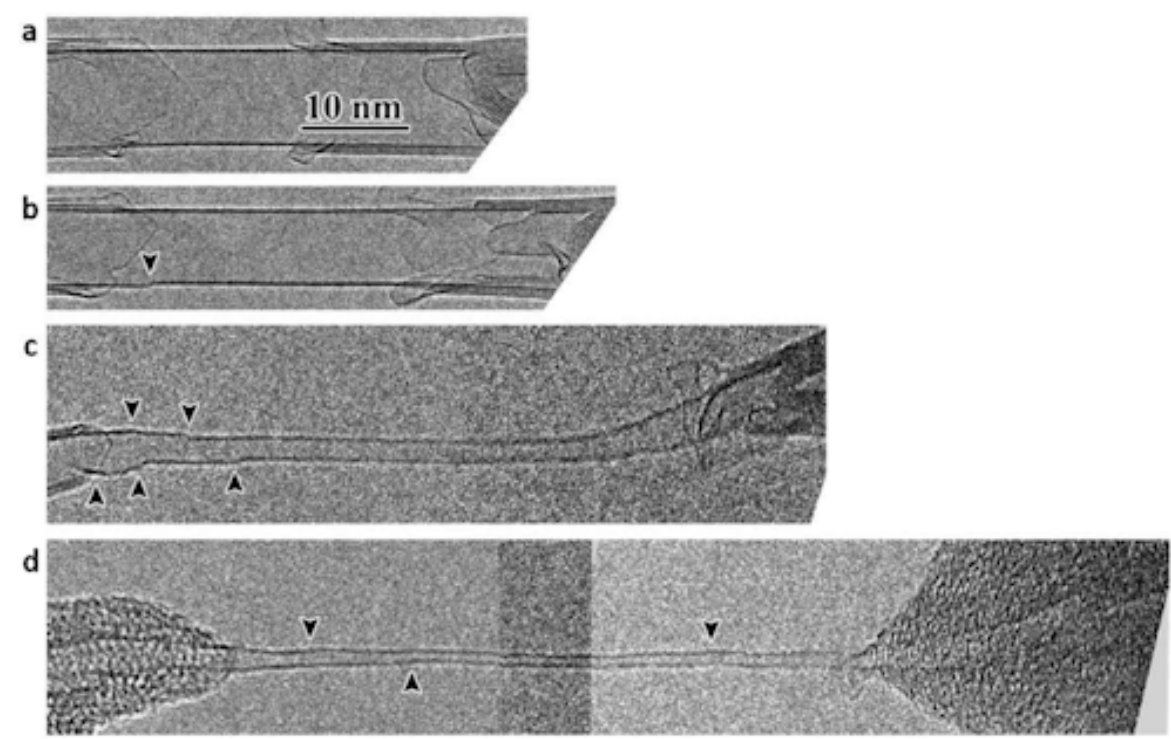

Fig. 4 (a) - (d) HRTEM images showing superplastic tensile elongation of individual singlewalled carbon nanotube under a bias of $2.3 \mathrm{~V}$, from the work of Huang et al. [13].

In a paper entitled "Shrinking a carbon nanotube", published a short time after the Huang work, Zettl and colleagues showed how a combination of Joule heating and electron irradiation could be used to dramatically reduce the diameter of carbon nanotubes [15]. Once again, this work involved the use of in situ TEM. Arc-grown nanotubes were positioned between electrical contacts and a voltage was applied. At voltages above of $2 \mathrm{~V}$, shrinkage 
was observed, and this continued until fracture occurred, as shown in Fig. 5. The precise voltages required depended on the intensity of the electron beam. The authors suggested that the shrinkage was due to a combination of knock-on damage induced by the $100 \mathrm{keV}$ TEM electron beam and Joule heating and electromigration produced by the electric current. The phenomenon appears similar to that observed by Huang et al., although in this case the tubes were not stressed.

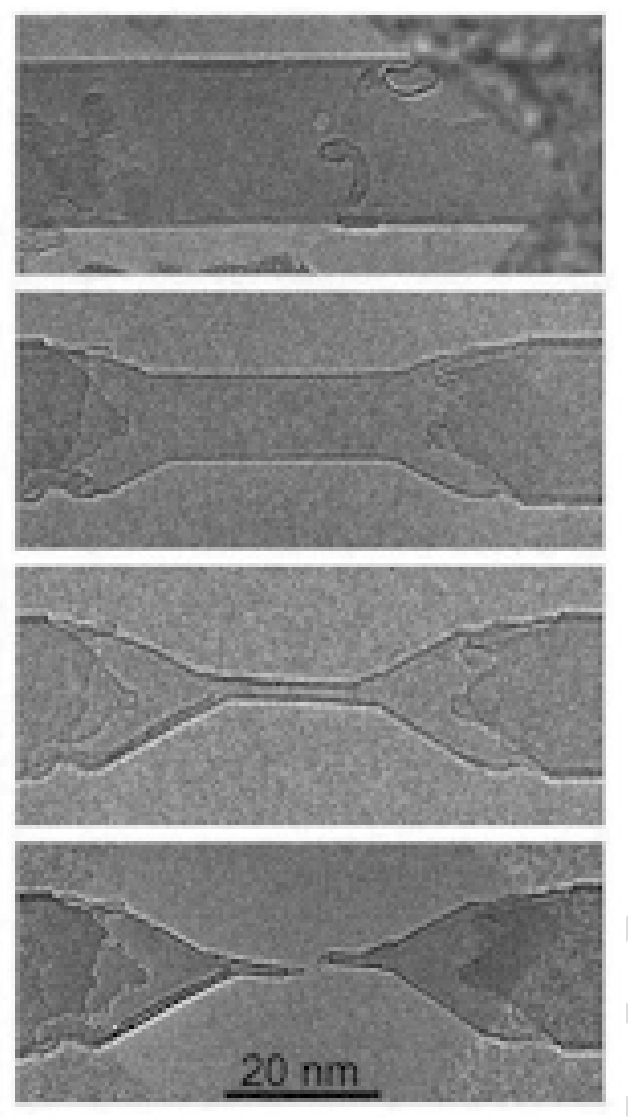

\begin{tabular}{|ll|}
\hline Fig. 5 & In situ TEM images of \\
& double-walled nanotube as \\
& it shrinks and eventually \\
& fractures as a result of \\
& passage of electric current \\
& and exposure to electron \\
& beam, adapted from \\
& reference 15. \\
\hline
\end{tabular}

Another spectacular demonstration of how an electric current can be used to engineer carbon nanomaterials was described by Jin et al. in 2008 [16]. Prior to this work, the idea of joining carbon nanotubes together to create junctions which might form the basis for nanoelectronic devices had been widely discussed from a theoretical standpoint, but few examples of "plumbing" of individual tubes had been reported. Jin and colleagues showed that Joule heating could be used to connect two nanotubes, with similar diameters, in a controlled way. A piezo-driven stage was used to manipulate the nanotubes inside a TEM, and this enabled the tubes to be brought into close proximity. A current was then passed to produce the junction, as shown in Fig. 6. The authors suggested that the mechanism of the joining process involved a series of Stone-Thrower-Wales transformations and other bond rearrangements, the activation energy for which is of the order of a few eVs. They concluded that Joule 
heating alone was not sufficient to produce the transformations and that electromigration must also have played a role.
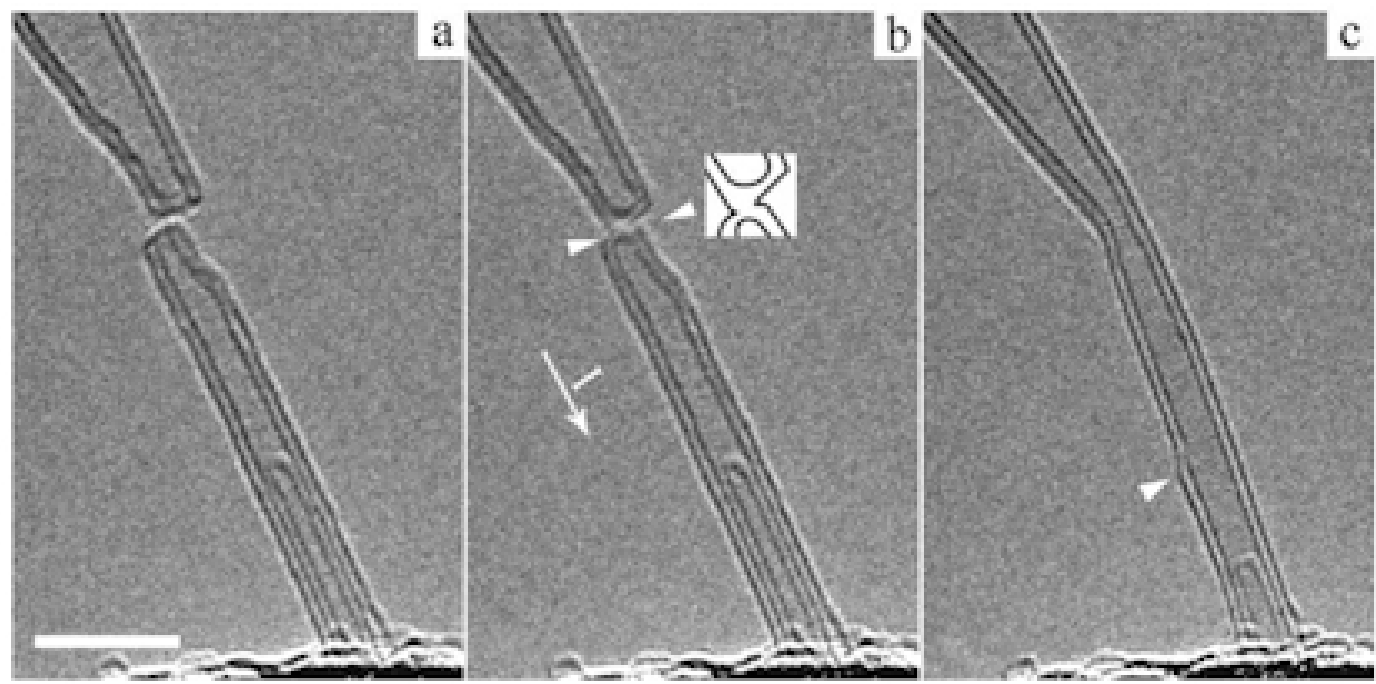

Fig. 6 Micrographs showing "plumbing" together of two DCWNTs to produce a single nanotube, from work by Jin et al. [16]. In (a) the two tubes can be seen being brought together. In (b) a current of $\sim 20 \mu \mathrm{A}$, at $1.7 \mathrm{~V}$, is passed and a junction begins to form as illustrated in the sketch. (c) Shows the final, continuous tube. Scale bar $5 \mathrm{~nm}$.

Since the work by Jin and colleagues, a few other groups have demonstrated the joining of carbon nanotubes by the passage of a current [e.g. ref. 17] but the technique has not been widely used, owing to its experimental difficulty.

\subsection{Graphene}

Electrical breakdown has not yet been extensively used to engineer the structure of graphene, but several studies of the breakdown of graphene ribbons have been reported. One of the first of these was published by Murali and colleagues in 2009 [5]. These workers created graphene nanoribbons with widths down to $16 \mathrm{~nm}$ using electron beam lithography, and current was passed through these until breakdown occurred. Breakdown current densities of approximately $10^{8} \mathrm{~A} / \mathrm{cm}^{2}$ were found, as mentioned above. Scanning electron microscope (SEM) images showed that breakdown occurred in the centre of the ribbons, which the authors believed pointed to Joule heating as the most likely mechanism of breakdown.

Bockrath and colleagues used pulsed electrical breakdown to create narrow gaps in suspended single- and few-layer graphene devices [18]. They then demonstrated that the junction resistance could be controlled by the application of voltage pulses, with $4 \mathrm{~V}$ 
corresponding to an $\mathrm{ON}$ pulse that decreased the device resistance and $8 \mathrm{~V}$ corresponding to an OFF pulse that increased the resistance. Scanning electron microscopy imaging of the device showed that the gap in the ON and state was smaller than in the OFF state. It was suggested that the switching mechanism involved atomic movement and/or chemical rearrangements.

The two studies mentioned so far were carried out in a vacuum. Nanometer-sized gaps in graphene can also be produced by passing a current in air, or under a small pressure of oxygen, in which case the term "electroburning" is sometimes used. One of the first demonstrations of this was reported by Prins et al. in 2011 [19]. These workers created gaps 1 $-2 \mathrm{~nm}$ wide in few-layer graphene ribbons using a feedback controlled electroburning technique. Feedback control over the process was necessary to control the gap size: in the absence of feedback the process is self-accelerating and results in relatively large gaps. In later work, Nef and colleagues described the fabrication of sub-5 nm gaps in single-layer graphene by carrying out electroburning under a small oxygen pressure under a vacuum of $\approx 10^{-5}$ mbar [20]. They found that using these conditions gave them more control than carrying out the process in ambient air. An interesting study of electroburning of graphene was described by Sadeghi et al. [21]. They found that the conductance of electroburnt junctions showed a surprising increase just before breaking. They attributed this to the formation of a picoscale current path formed from a single $\mathrm{sp}^{2}$ bond.

There is growing interest in the use of electroburning to engineer graphene structures, although achieving the delicate degree of control required is clearly a challenge.

\subsection{Disordered carbon}

Relatively little work has been carried out on the effect of passing an electric current on the structure of disordered carbons. One of the few such studies was described by Huang et al. in 2006 [22]. In this work, a current was passed through disordered carbon nanowires inside a TEM, resulting in the formation of graphitized structures, some of which were tubular in form (see Fig. 7). However, it is not clear whether the electric field was responsible for the formation of tubular structures, or whether this was simply a consequence of the extended shapes of the original amorphous carbon precursors. Marks et al. carried out a theoretical analysis of the evolution of amorphous precursors into carbon nanostructures and showed that for a one-dimensional amorphous precursor the final structure was tubular [23]. This was 
confirmed in subsequent work by the same group [24]. Since there is little evidence that amorphous "nanorods" are preferentially formed in the arc, Huang's work may not provide a realistic model of the formation of nanotubes by arc-discharge. However, the synthesis of nanotubes from amorphous carbon deposited onto InAs nanowires has been demonstrated [25].

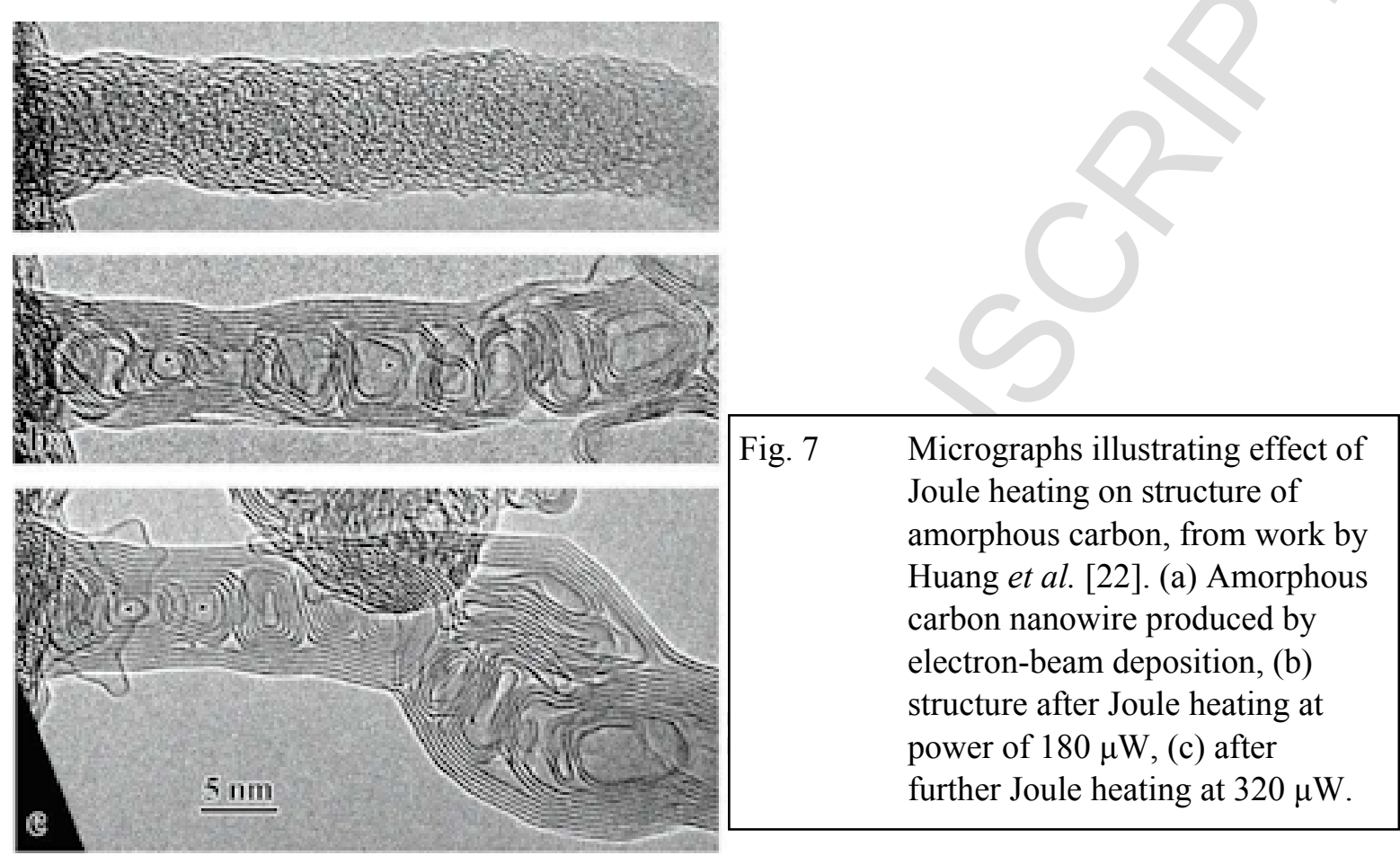

\section{Effects apparently due to electrostatic charging}

\subsection{Structural transformation induced by electric field}

Three studies published in 2009 described dramatic structural transformations induced by the application of an electric field to graphite [26 - 28]. These transformations appeared to be of a different kind to those previously observed. Two of these studies involved the in situ heating of few-layer graphite "nanoribbons" inside a TEM. In both cases it was found that the graphite ribbons evolved into complex structures which largely seemed to be made up of single-layer graphene. Examples of the transformed structures, taken from both papers, are shown in Fig. 8. The third study, by the present author, involved a TEM 

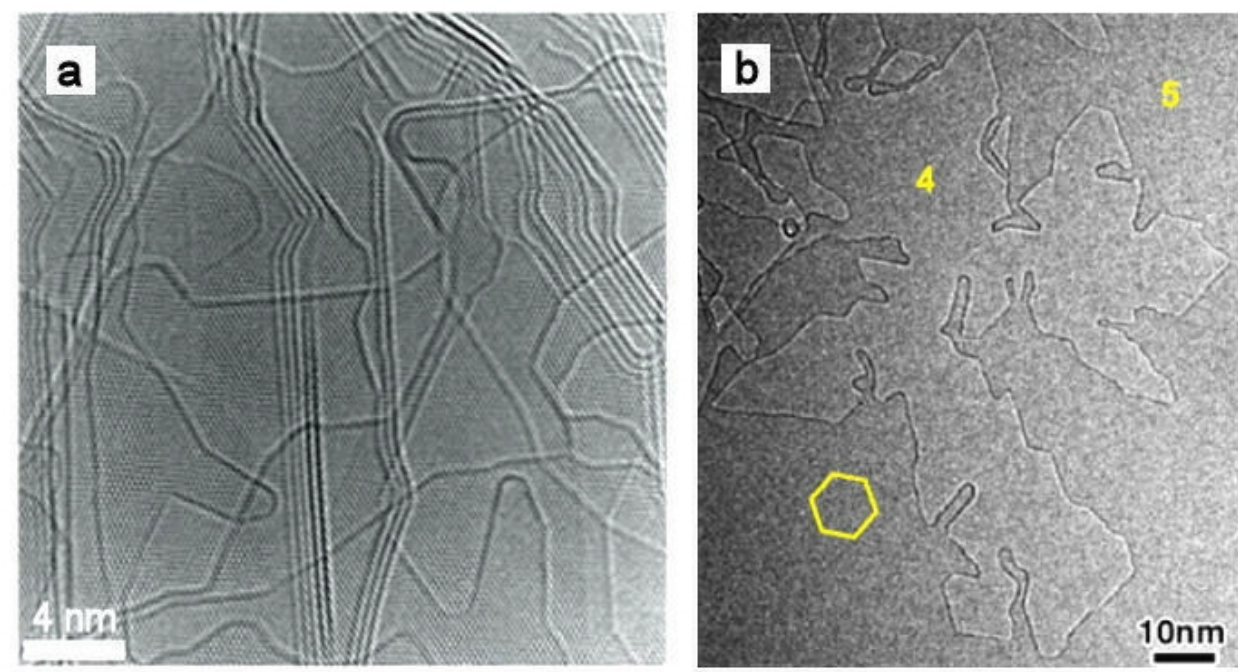

Fig. 8 (a) Structure produced by in situ Joule heating of graphitic nanoribbon inside a TEM, from the work of Jia et al. [26]. (b) Structure produced in a similar way by Huang et al. [27]. The numbers 4 and 5 in this figure indicate regions which the authors believe to contain 4 and 5 bilayers, respectively. The hexagon marks the orientation of the 6 equivalent $\{1100\}$ planes.
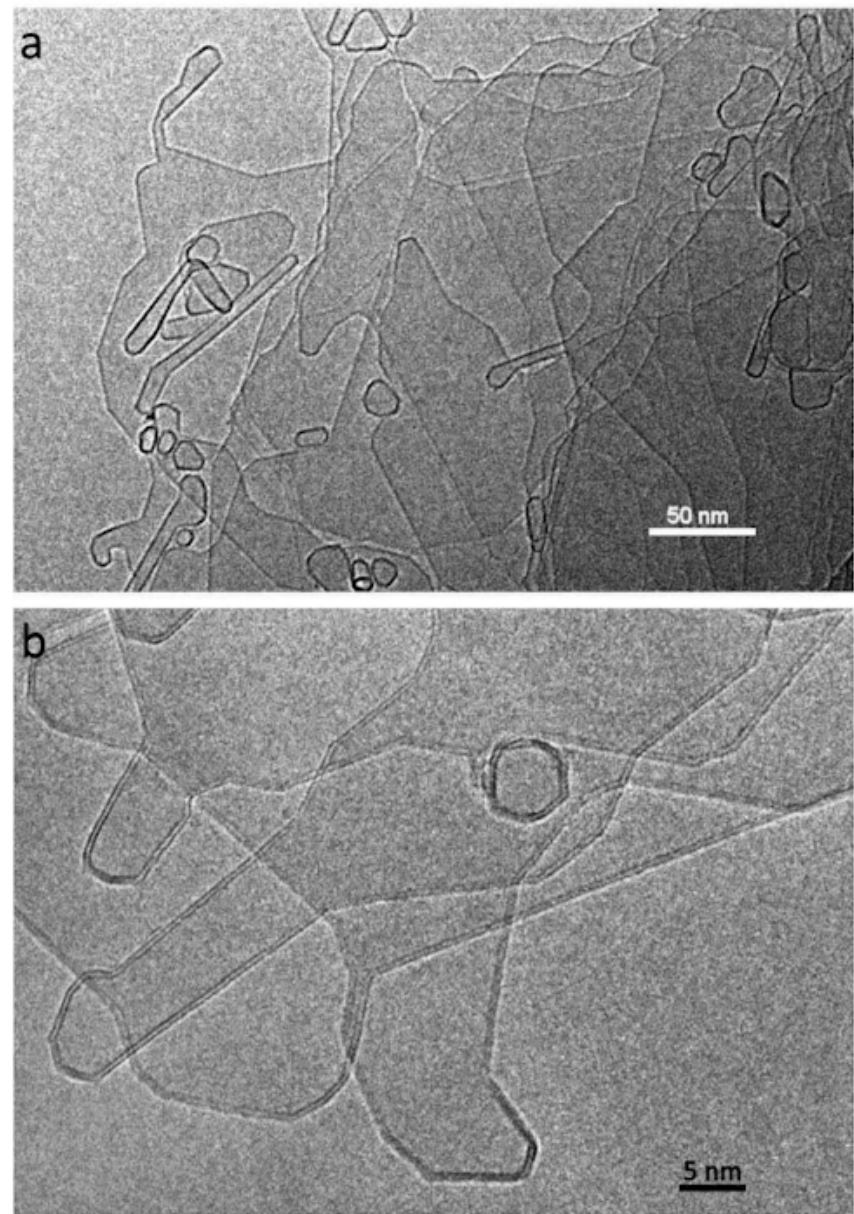

\begin{tabular}{|ll}
\hline Fig. 9 & Micrographs showing \\
& carbon material produced by \\
& exposing graphite to an \\
& electric field at high \\
& temperature. (a) Moderate \\
& magnification image, (b) high \\
& magnification image showing \\
& bilayer structure.
\end{tabular}


investigation of bulk graphite following exposure to an electric field. In this case the transformed carbon mainly consisted of bilayer graphene structures. Examples of the kind of structures observed are shown in Fig. 9. Following this initial work, several further studies of the phenomenon have been published [29 - 36]. There is disagreement about the nature of the transformation. One view is that the phenomenon involves sublimation and edge reconstruction of essentially flat graphitic structures [26, 27, 33 - 36]. Thus, Huang and colleagues $[27,33,34]$ proposed that the morphologies observed in the transformed material could be understood in terms of a fractal-like structure produced by sublimation and reconstruction, while Jia et al. [26, 36] highlighted the importance of zig-zag or armchair edges in stabilizing the transformed structures. A quite different hypothesis has been put forward by the present author and colleagues [29 - 32], namely that the transformation actually involves a change from a flat to a three-dimensional structure.

There are a number of reasons for believing that the transformed structures are threedimensional and hollow rather than flat. These include the observation that small nanoparticles or nanotubes are sometimes seen encapsulated inside larger structures, as shown in Fig. 10 (a) and that nanotubes are often found to be seamlessly joined to the thin shells, as in Fig. 10 (b). Direct evidence for the idea that the structures are three-dimensional has been obtained by using a combination of high-angle annular dark-field imaging and electron energy loss spectroscopy in the scanning transmission electron microscope [31]. A discussion of the possible mechanism of the transformation of flat graphite into a threedimensional structure is given in the next section.
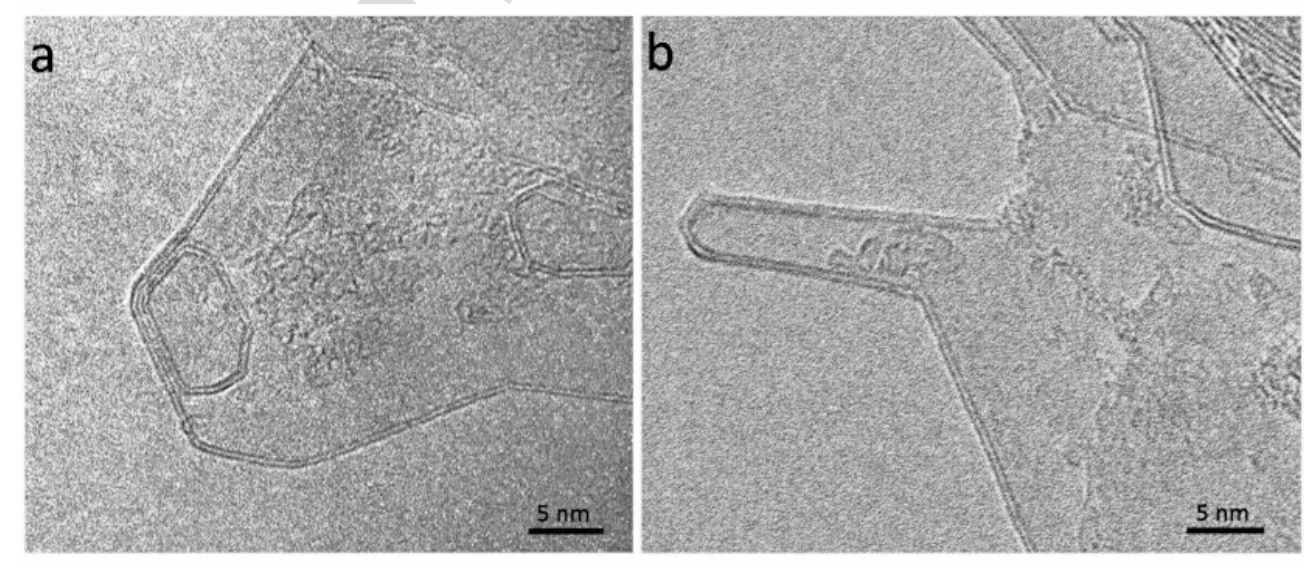

Fig. 10 Structures in graphite transformed by exposure to an electric field. (a) Bilayer graphene structure, apparently hollow with bilayer nanoparticle inside, (b) bilayer nanotube joined to larger graphene structure [32]. 
Recently, an interesting study of the effect of an electric field on the structure of carbon nanofibers has been published by Wang et al. [37]. The nanofibres were synthesized using chemical vapor growth, a process that tends to produce fibres with a relatively low degree of graphitization. Their work was primarily concerned with improving the graphitization of the fibres by the passage of a current, and it was shown that the conductivity could be increased
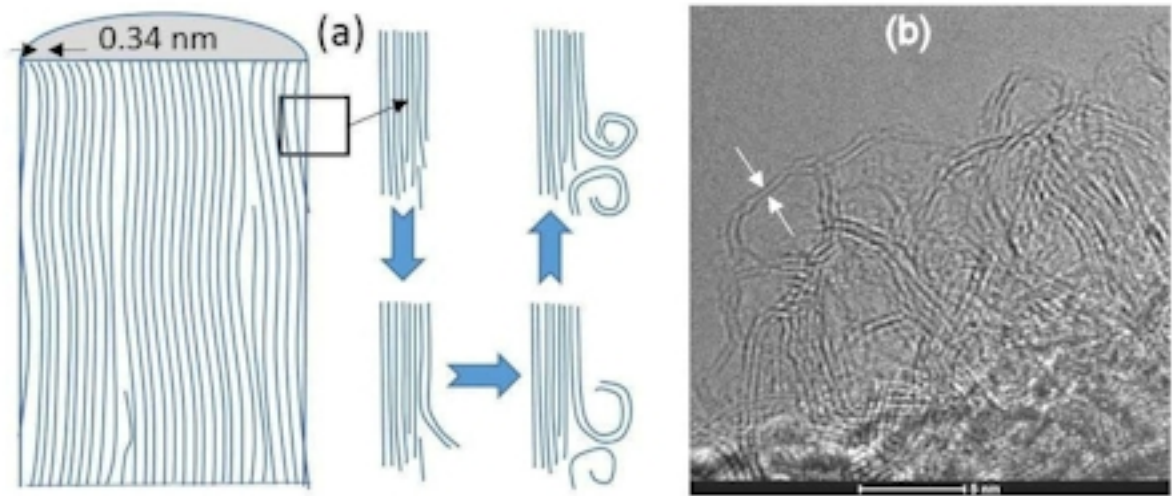

Fig. 11 (a) Schematic illustration of electrostatic exfoliation mechanism for surface layers of carbon nanofibres, (b) micrograph showing structure of exfoliated carbon. From work by Wang et al. [37].

by 3 orders of magnitude by employing current densities up to $10^{6} \mathrm{~A} / \mathrm{cm}^{2}$. Higher current densities led to the exfoliation of mostly bilayer graphene flakes from the skin of the graphitic nanofibers, as shown in Fig. 11. The formation of bilayer graphene structures is reminiscent of the work on the transformation of bulk graphite described above. Wang and colleagues suggested a mechanism in which individual layers in the fibres accumulated electrostatic charge, and this charge accumulation led to the exfoliation of the surface layers. This would seem to be a highly plausible explanation for the phenomenon observed by Wang et al., as well as for the other structural transformations described in this section.

\subsection{The mechanism of structural transformation}

This section considers in more detail the mechanism of the electric field-induced transformations described above. It is assumed that the process involves an evolution from a flat to a three-dimensional structure rather than sublimation and edge reconstruction of flat graphene. In discussing mechanism it is helpful to consider the edge structure of graphite. It is well established that graphite planes often have "closed"' edges, so that the layers resemble folded sheets [38 - 42], as illustrated in Fig. 12 (a). The transformations reported in the previous section may simply begin with an "opening'" of the layers, as shown 
schematically in Fig. 12 (b). Supporting evidence for this mechanism, is provided by images showing the early stages of the process, as shown in Fig. 12 (c). The question arises, therefore, of why the application of an electric field should result in the opening of the graphite layers. It was pointed out by the present author that the phenomenon is reminiscent of the separation of gold leaves in an electroscope [32], although it was not clear whether this a $\quad b$
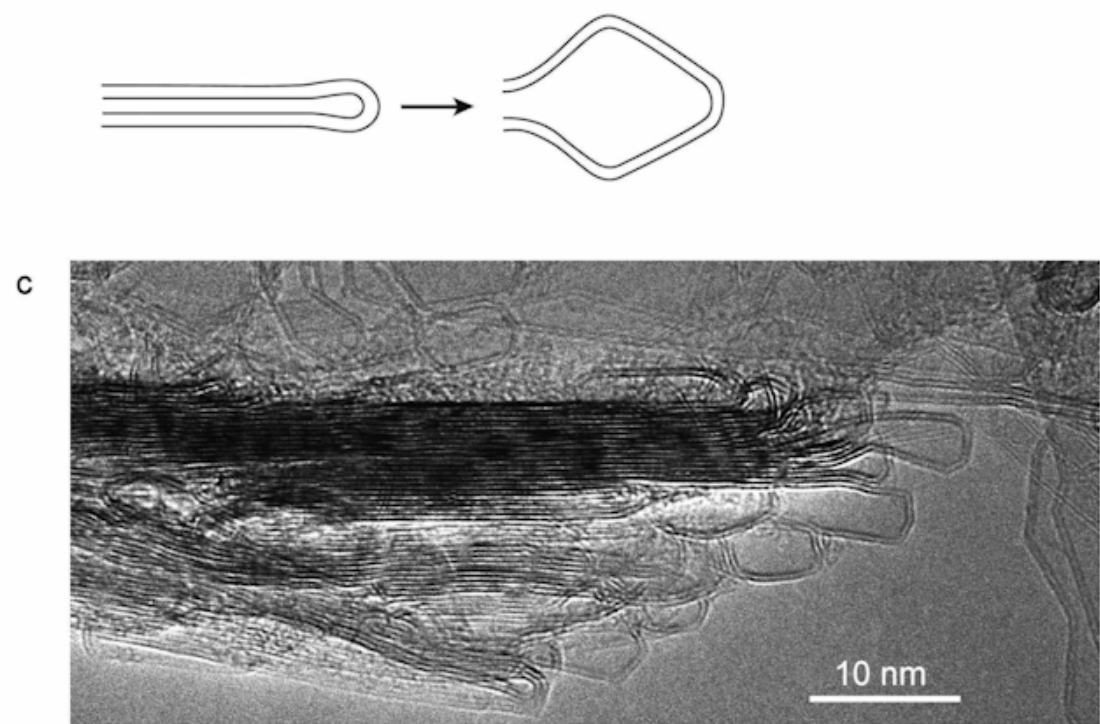

Fig. 12 (a) and (b) Schematic illustration showing suggested mechanism for transformation of folded graphene sheets into hollow structure, (c) HRTEM image of graphitic region edge-on, apparently showing early stages of transformation [31].

was relevant. As noted above, Wang et al. have suggested that an analogous process is indeed involved, in which charge accumulates in the graphene layers, leading to repulsion between the layers and separation. This idea is supported by theoretical work which shows that an external field applied perpendicular to a pair of graphene layers can drive the system to a unstable state where the layers are decoupled and can be easily separated [43, 44].

One of the most interesting features of graphite transformed by an electric field is the presence of nanotubes joined to graphene shells, as shown in Fig. 10 (b). A detailed analysis of these junctions has been carried out and has shown that the junction angles are not random but fall close to multiples of $30^{\circ}$ [45]. It was demonstrated that connections with these angles are the only ones which are consistent with the symmetry of the hexagonal lattice, and molecular models showed that a continuous lattice requires the presence of large carbon rings at the junction. The possible formation mechanism of the junctions was also discussed, and it 
was suggested that the process might be initiated at the pentagonal rings which are believed to occur where a zigzag edge meets an armchair edge.

\subsection{Electrostatic exfoliation}

The exfoliation of graphite to produce graphene has been the subject of a huge amount of work [46], but there have been relatively few studies on the use of electric fields to induce exfoliation. Liang and co-workers were among the first to explore this approach. In 2009 they described the use of electrostatic exfoliation to deposit patterned few-layer graphene onto a substrate [47]. Their method involved firstly creating patterns on the surface of a highly oriented pyrolytic graphite (HOPG) disc using lithographic techniques. A feature of the pattern was then brought into contact with an $\mathrm{SiO}_{2} / \mathrm{Si}$ substrate and a voltage was applied between the HOPG template and the substrate. This resulted in exfoliation of graphene flakes from the HOPG, and attachment to the substrate. Using this technique the authors demonstrated the exfoliation/printing of $18 \mathrm{~nm}$ wide graphene "nanolines", which could be used to fabricate nano transistors.

Two recent studies have explored different methods of electric field-induced exfoliation. Rubio-Verdú and colleagues showed that a scanning tunneling microscopy (STM) tip could be used to locally exfoliate HOPG by applying an electrostatic force at the edge of a terrace, forming triangular flakes [48]. Macroscopic exfoliation of graphite was demonstrated by Gao et al. [49]. In this work a voltage of around $20 \mathrm{kV}$ was applied to graphite rods held under water. This resulted in an explosion which produced a variety of carbon species including few-layer and monolayer graphene sheets. These interesting studies suggest that the technique of electrostatic exfoliation deserves further research.

\subsection{Graphene origami}

The concept of graphene origami was first put forward in a 1995 paper by Ebbesen and Hiura [50]. In this paper, which anticipated the "discovery" of graphene by 9 years, scanning probe microscopy was used to create folds in graphene layers on the surface of highly ordered pyrolytic graphite. While this represented a significant technical feat, the resulting structures were still essentially two-dimensional. In 2014 Zhu and Li showed how, in theory, an electric field could be used to create three-dimensional graphene "nanocages" [51]. Their simulation began with a double cross-shaped graphene flake, as shown in Fig. 13 (a). Hydrogen atoms were then added around the edges of the flake, and at the junctions between the square 
sections. They then showed that the structure could be reversibly closed and opened by the application of an external electric field, due to the polarization of carbon atoms. The closed nanocage is shown in Fig. 13 (b). It was suggested that the cages could be used for highdensity hydrogen storage.

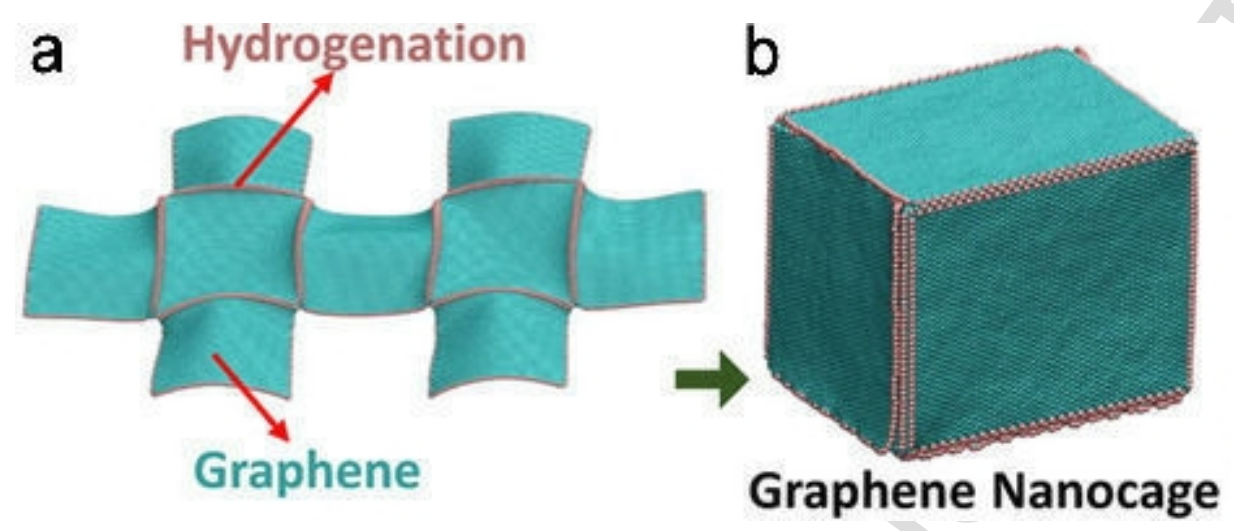

Fig. 13 Illustration of folding of hydrogenated graphene structure to form closed nanocages, induced by electric field, from the work of Zhu and $\mathrm{Li}$ [51].

\section{Experiments with doped and filled nanotubes}

In a study published in 2006 [52], Peng et al. prepared amorphous carbon nanowires using a similar method to that of Huang et al. [22], and deposited Fe particles onto the wires. A current was then passed through these structures and this caused the Fe particles to move along the nanowires, resulting in graphitization. The breakdown current density of the original amorphous nanowires was found to be $2.2 \times 10^{5} \mathrm{~A} / \mathrm{cm}^{2}$, but this increased to $4.6 \times 10^{8} \mathrm{~A} / \mathrm{cm}^{2}$ for the graphitized nanowires. This figure is comparable to the values found for nanotubes and graphene ribbons (see above), suggesting that the graphitized nanowires could be useful in nanoelectronic applications.

Grobert and colleagues have explored the effect of Joule heating on nitrogen and borondoped carbon nanotubes and on filled nanotubes [53 - 55]. In the first of these studies [53], $\mathrm{N}$-doped carbon nanotubes, grown catalytically, were subjected to Joule heating inside a TEM. The initial N-MWCNTs were rather disordered and often had a bamboo-like structure. Figure 14 shows the effect of passing a current of $47 \mu \mathrm{A}$ at $2000 \mathrm{mV}$ through an individual tube. It can be seen that the structure becomes progressively more ordered. There is also evidence for the formation of single-layer material which resembles some of the "three- 
dimensional graphene" structures discussed in Section 3.1 above. At sufficiently high current densities the nanotubes fractured, often leaving conical tips. The structure of these tips appeared to be related to the nature of the "compartments" in the original tubes.

As well as imaging the N-MWCNTs, Grobert et al. used electron energy loss spectroscopy (EELS) to determine the nitrogen content of the tubes before and after Joule heating. The asproduced tubes were found to contain approximately $3 \% \mathrm{~N}$, but the $\mathrm{N}$ was completely removed by the passage of current. The restructuring and loss of $\mathrm{N}$ resulted in a significant increase in the conductivity of the tubes.
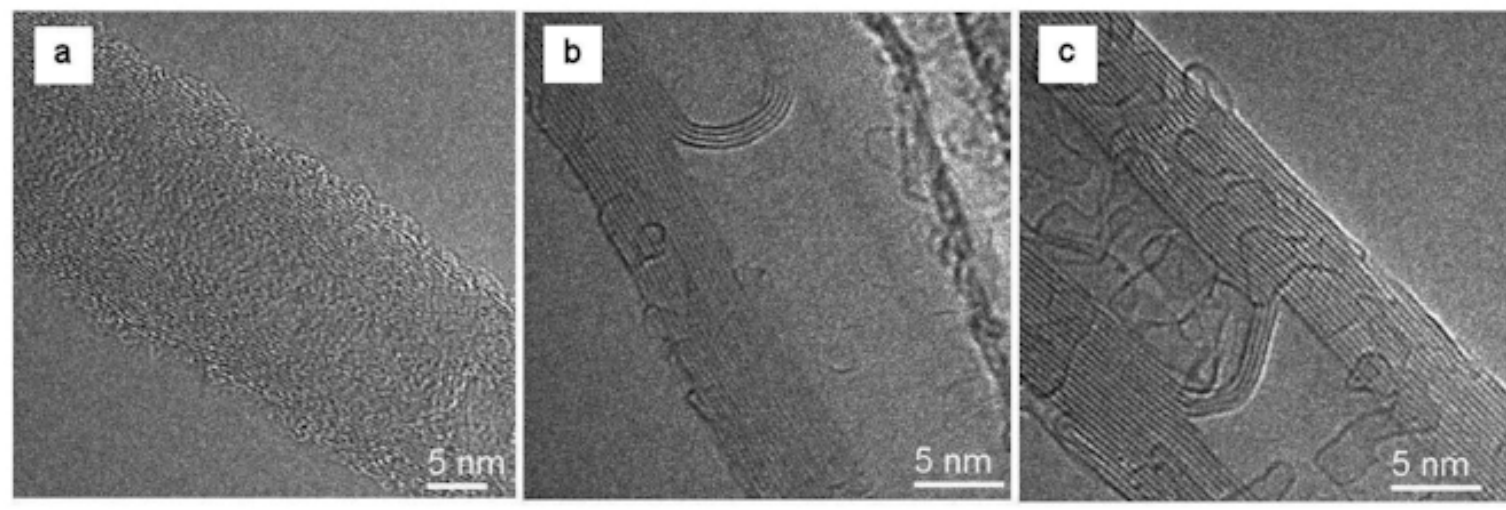

Fig. 14 Images showing nitrogen-doped MWCNT before (a) and during (b), (c), the application of current, from work by Grobert and colleagues [53].

The same group carried out a similar study of boron-doped MWCNTs [54]. Again the passage of current resulted in restructuring of the tubes, and in loss of the dopant, although some B was retained in the tubes after Joule heating. As in the case of the $\mathrm{N}$-doped tubes, the passage of current resulted in changes in the electrical behaviour of the B-MWCNTs, and high current densities resulted in fracture of the tubes. Both of these studies show that care needs to be taken to limit the maximum current density if doped-MWCNTs are to be used in electronic applications.

Some interesting work has been carried out on the passage of a current on filled carbon nanotubes. Costa and colleagues used a nanomanipulator inside a TEM to make connections between electrodes and filled tubes, and then showed that passing a current could induce the transport of the filling along the tube [56], an effect similar to that previously reported by 
Peng et al. [52]. In subsequent work [57] the same group showed how experiments with filled tubes could be used to determine the temperatures which were generated by the passage of current. Multiwalled nanotubes filled with a low vapour pressure material, namely $\mathrm{Zn}_{0.92} \mathrm{Ga}_{0.08} \mathrm{~S}$, were used in this work. Two different configurations were used: one with the tube connected at both ends, and one with an electrode connected to a side wall. In both cases the passage of a current resulted in the expulsion of the filling material. Figure 15 shows the effect of Joule heating on a nanotube connected at both ends. Initially, sulphide is removed from the area close to the Au cathode. Then a "hotspot" develops further along the tube, creating a gap in the filling as sulphide vaporises (Fig. 15 (b)). The two segments are then progressively consumed by the expansion of the hotspot (Fig. 15 (c)). The segment fronts acted as markers for the sulphide sublimation temperature $(928 \mathrm{~K})$. Further
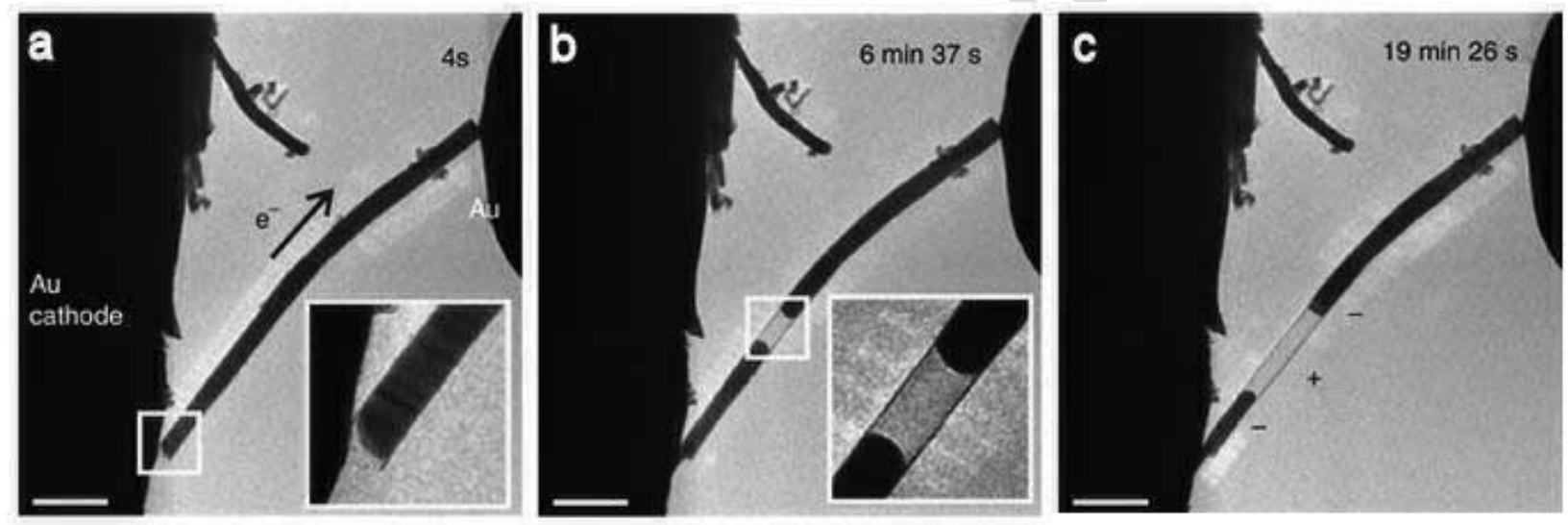

Fig. 15 Micrographs illustrating electrically induced removal of $\mathrm{Zn}_{0.92} \mathrm{Ga}_{0.08} \mathrm{~S}$ from inside of
MWCNT. Plus and minus signs in (c) refer to temperatures above and below the
sublimation point of the core. Scale bar $0.5 \mu \mathrm{m}$. From work by Costa et al. [57].

detailed studies of filled tubes enabled Costa et al. to determine, for the first time, the radial heat distribution inside CNTs.

In more recent work, the Grobert group has shown how the structure of $\mathrm{Cr}_{2} \mathrm{O}_{3}$ encapsulated inside MWCNTs can be transformed by Joule heating of the filled tubes [55].

\section{Discussion}

A very large amount of work has been carried out on the modification of carbon nanomaterials using electricity and this article is not intended to be an exhaustive review. Instead, an attempt has been made to highlight some of the most significant and striking 
results in this field, and to distinguish between the effect of passing an electric current and the effect of the application of an electrostatic charge. The passage of current has been used to create gaps in nanotubes and graphene by inducing breakdown. The opposite process, i.e. the plumbing together of nanotubes by passing a current through them, has also been demonstrated. While many of these laboratory demonstrations are extremely impressive, there is still a long way to go before similar techniques can be used to create useful electronic devices containing thousands or millions of connections.

The discovery of "superplastic carbon nanotubes" by Huang et al. [13] created wide interest. The demonstration that carbon nanotubes can be stretched by almost $280 \%$ when a current is passed through them at high temperatures came as a surprise, given that the room temperature breaking strains of carbon nanotubes are typically around $10 \%$. It is not clear whether the remarkable ductility observed in these experiments was a result of the high temperature, the electric current, or both. More work on this phenomenon would be welcome.

There has been growing interest recently in the massive restructuring of carbon materials which can be induced by the application of an electric field to graphite or to few-layer graphene. This phenomenon was discovered independently by three groups in 2009 [26 - 28], and involves the formation of a carbon material with a highly irregular edge morphology, with many re-entrant structures and unusual features including nanotubes seamlessly joined to larger graphene regions. The precise mechanism of this restructuring is still a matter of debate. It has been argued here, and in previous papers, that the process involves an evolution from a flat to a three-dimensional structure rather than sublimation and edge reconstruction of flat graphene, as has been suggested by other authors. It has also been argued that this transformation is initiated when charge accumulates in the graphene layers, leading to repulsion between the layers and separation, a suggestion that was first made explicitly by Wang et al. [37]. However, this is far from full explanation of the phenomenon. One of the most remarkable aspects of the transformation is the extreme fluidity of the carbon structures when exposed to an electric field at high temperatures. This can be seen graphically in the videos which accompany reference 27 . In some ways this resembles the superplasticity observed by Huang and colleagues; neither phenomenon is well understood.

It is clear that the application of an electric field can promote dramatic restructuring of carbon materials, but the transformations produced in this way are uncontrolled, creating essentially 
random structures. This contrasts with the very precise control, on the nm scale, which can be achieved by electrical breakdown. Is it possible to envisage a way in which the more controlled application of a field could be used to engineer defined, three-dimensional carbon structures? The intriguing theoretical work by Zhu and Li on "graphene origami" shows one way in which an electric field could be used to create 3D graphene structures with defined shapes [51]. While carrying out graphene origami experimentally may be beyond our present capabilities, theoretical work of this kind shows that the application of electric fields could have great potential for the controlled manipulation of graphene on the nanoscale.

\section{Acknowledgments}

I thank Ferry Prins, Yunqi Liu, Zhifeng Ren, Nigel Marks for helpful comments.

\section{References}

[1] G. Collin, On the history of technical carbon, CFI-Ceramic Forum International 77 (2000) 28-35.

[2] W. Krätschmer, L.D. Lamb, K. Fostiropoulos, D.R. Huffman, Solid $\mathrm{C}_{60}$ : a new form of carbon, Nature 347 (1990) 354-358.

[3] S. Iijima, Helical microtubules of graphitic carbon, Nature 354 (1991) 56-58.

[4] A. Javey, P.F. Qi, Q. Wang, H.J. Dai, Ten- to 50-nm-long quasi-ballistic carbon nanotube devices obtained without complex lithography, Proc. Nat. Acad. Sci. U.S.A. 101 (2004) 13408-13410.

[5] R. Murali, Y. Yang, K. Brenner, T. Beck, J.D. Meindl, Breakdown current density of graphene nanoribbons, Applied Physics Letters 94 (2009) 243114.

[6] P.G. Collins, M. Hersam, M. Arnold, R. Martel, P. Avouris, Current saturation and electrical breakdown in multiwalled carbon nanotubes, Physical Review Letters 86 (2001) 3128-3134.

[7] P.G. Collins, M.S. Arnold, P. Avouris, Engineering carbon nanotubes and nanotube circuits using electrical breakdown, Science 292 (2001) 706-709.

[8] P. Qi, A. Javey, M. Rolandi, Q. Wang, E. Yenilmez, H. Dai, Miniature organic transistors with carbon nanotubes as quasi-one-dimensional electrodes, J. Amer. Chem. Soc. 126 (2004) 11774-11775.

[9] K. Tsukagoshi, I. Yagi, Y. Aoyagi, Pentacene nanotransistor with carbon nanotube electrodes, Applied Physics Letters 85 (2004) 1021-1023. 
[10] D. Wei, Y. Liu, L. Cao, Y. Wang, H. Zhang, G. Yu, Real time and in situ control of the gap size of nanoelectrodes for molecular devices, Nano Letters 8 (2008) $1625-1630$.

[11] J.Y. Huang, S. Chen, S.H. Jo, Z. Wang, D.X. Han, G. Chen, M.S. Dresselhaus, Z.F. Ren, Atomic-scale imaging of wall-by-wall breakdown and concurrent transport measurements in multiwall carbon nanotubes, Physical Review Letters 94 (2005) 236802 .

[12] C. Jin, K. Suenaga, S. Iijima, Direct evidence for lip-lip interactions in multi-walled carbon nanotubes, Nano Research 1 (2008) 434-439.

[13] J.Y. Huang, S. Chen, Z.Q. Wang, K. Kempa, Y.M. Wang, S.H. Jo, G. Chen, M. S. Dresselhaus, Z. F. Ren, Superplastic carbon nanotubes, Nature 439 (2006) 281.

[14] F. Ding, K. Jiao, Y. Lin, B.I. Yakobson, How evaporating carbon nanotubes retain their perfection?, Nano Letters 7 (2007) 681-684.

[15] T.D. Yuzvinsky, W. Mickelson, S. Aloni, G.E. Begtrup, A. Kis, A. Zettl, Shrinking a carbon nanotube, Nano Letters 6 (2006) 2718-2722.

[16] C. Jin, K. Suenaga, S. Iijima, Plumbing carbon nanotubes, Nature Nanotechnology 3 (2008) 17-21.

[17] K. Asaka, M. Karita, Y. Saito, Joining of multiwall carbon nanotubes for the endcontact configuration by applying electric current, Materials Letters 65 (2011) 18321834.

[18] H. Zhang, W.Z. Bao, Z. Zhao, J.W. Huang, B. Standley, G. Liu, F.L. Wang, P. Kratz, L. Jing, M. Bockrath, C.N. Lau, Visualizing electrical breakdown and on/off states in electrically switchable suspended graphene break junctions, Nano Letters 12 (2012) $1772-1775$.

[19] F. Prins, A. Barreiro, J.W. Ruitenberg, J.S. Seldenthuis, N. Aliaga-Alcalde, L.M.K. Vandersypen, H.S.J. van der Zant, Room-temperature gating of molecular junctions using few-layer graphene nanogap electrodes, Nano Letters 11 (2011) 4607-4611.

[20] C. Nef, L. Pósa, P. Makk, W. Fu, A. Halbritter, C. Schönenberger, M. Calame, High-yield fabrication of nm-size gaps in monolayer CVD graphene, Nanoscale 6 (2014) 7249-7254.

[21] H. Sadeghi, J.A. Mol, C.S. Lau, G.A.D. Briggs, J. Warner, C.J. Lambert, Conductance enlargement in picoscale electroburnt graphene nanojunctions, Proceedings of the National Academy of Sciences of the United States of America 112 (2015) 2658-2663.

[22] J.Y. Huang, S. Chen, Z.F. Ren, G. Chen, M.S. Dresselhaus, Real-time observation of tubule formation from amorphous carbon nanowires under high-bias Joule heating, Nano Letters 6 (2006) 1699-1705. 
[23] R.C. Powles, N.A. Marks, D.W.M. Lau, Self-assembly of $\mathrm{sp}^{2}$-bonded carbon nanostructures from amorphous precursors, Phys. Rev. B 79 (2009) 075430.

[24] I. Suarez-Martinez, N.A. Marks, Amorphous carbon nanorods as a precursor for carbon nanotubes, Carbon 50 (2012) 5441-5449.

[25] K. Zheng, R. Shao, J. Wang, Z. Liao, N. Marks, P. Chen, W. Lu, X. Han, J. Zou, Fabrication of individual carbon nanotubes and their arrays in a transmission electron microscope, Carbon 100 (2016) 435-440.

[26] X.T. Jia, M. Hofmann, V. Meunier, B.G. Sumpter, J. Campos-Delgado, J.M. RomoHerrera, H. Son, Y.P. Hsieh, A. Reina, J. Kong, M. Terrones, M.S. Dresselhaus, Controlled formation of sharp zigzag and armchair edges in graphitic nanoribbons, Science 323 (2009) 1701-1705.

[27] J.Y. Huang, F. Ding, B.I. Yakobson, P. Lu, L. Qi, J. Li, In situ observation of graphene sublimation and multi-layer edge reconstructions, Proceedings of the National Academy of Sciences of the United States of America 106 (2009) 1010310108.

[28] P.J.F. Harris, Ultrathin graphitic structures and carbon nanotubes in a purified synthetic graphite, J. Physics: Condens. Matter 21 (2009) 355009.

[29] P.J.F. Harris, Structural transformation of graphite by arc-discharge, Philosophical Magazine 91 (2011) 2355-2363.

[30] P.J.F. Harris, Hollow structures with bilayer graphene walls, Carbon 50 (2012) 3195 3199.

[31] P.J.F. Harris, T.J.A. Slater, S.J. Haigh, F.S. Hage, D.M. Kepaptsoglou, Q.M. Ramasse, R. Brydson, Bilayer graphene formed by passage of current through graphite: evidence for a three-dimensional structure, Nanotechnology 25 (2014) 465601.

[32] P.J.F. Harris, Structural transformation of natural graphite by passage of an electric current, Carbon 107 (2016) 132-137.

[33] L. Qi, J.Y. Huang, J. Feng, J. Li, In situ observations of the nucleation and growth of atomically sharp graphene bilayer edges, Carbon 48 (2010) 2354-2360.

[34] J. S. Qi, J. Y. Huang, J. Feng, D. N. Shi and J. Li, The possibility of chemically inert, graphene-based all-carbon electronic devices with $0.8 \mathrm{eV}$ gap, ACS Nano 5 (2011) 3475-3482. 
[35] A. Barreiro, F. Börrnert, M.H. Rümmeli, B. Büchner, L.M.K. Vandersypen, Graphene at high bias: Cracking, layer by layer sublimation, and fusing, Nano Letters 12 (2012) 1873-1878.

[36] E. Cruz-Silva, X.T. Jia, H. Terrones, B.G. Sumpter, M. Terrones, M.S. Dresselhaus, V. Meunier, Edge-edge interactions in stacked graphene nanoplatelets, ACS Nano 7 (2013) 2834-2841.

[37] B. Wang, D.E. Wolfe, M. Terrones, M.A. Haque, S. Ganguly, A.K. Roy, Electrographitization and exfoliation of graphene on carbon nanofibers, Carbon 117 (2017) 201-207.

[38] P.R. Buseck, B.J. Huang, L.P. Keller, Electron-microscope investigation of the structures of annealed carbons, Energy and Fuels 1 (1987) 105-110.

[39] H. Murayama, T. Maeda, A novel form of filamentous graphite, Nature 345 (1990) 791-793.

[40] Y. Gogotsi, J.A. Libera, N. Kalashnikov, M. Yoshimura, Graphite polyhedral crystals, Science 290 (2000) 317-320.

[41] S.V. Rotkin, Y. Gogotsi, Analysis of non-planar graphitic structures: from arched edge planes of graphite crystals to nanotubes, Materials Research Innovations 5 (2002) 191-200.

[42] Z. Liu, K. Suenaga, P.J.F. Harris, S. Iijima, Open and closed edges of graphene layers, Physics Rev. Letters 102 (2009) 015501.

[43] E.J.G. Santos, E. Kaxiras, Electric-field dependence of the effective dielectric constant in graphene, Nano Letters 13 (2013) 898-902.

[44] E.J.G. Santos, Electric field effects on graphene materials, in M.V. Putz, O. Ori (Eds.), Exotic properties of carbon nanomatter, Springer, Netherlands (2015), pp. 383-391.

[45] P.J.F. Harris, I. Suarez, N.A. Marks, The structure of junctions between carbon nanotubes and graphene shells, Nanoscale 8 (2016) 18849.

[46] M. Cai, D. Thorpe, D.H. Adamson, H.C. Schniepp, Methods of graphite exfoliation, J. Materials Chemistry 22 (2012) 24992-25002.

[47] X. Liang, A.S.P. Chang, Y. Zhang, B.D. Harteneck, H. Choo, D.L. Olynick, S. Cabrini, Electrostatic force assisted exfoliation of prepatterned few-layer graphenes into device sites, Nano Letters 9 (2009) 467-472. 
[48] C. Rubio-Verdú, G. Sáenz-Arce, J. Martinez-Asencio, D.C. Milan, M. Moaied, J.J. Palacios, M. J. Caturla, C. Untiedt, Graphene flakes obtained by local electroexfoliation of graphite with a STM tip, Physical Chemistry Chemical Physics 19 (2017) 8061- 8068.

[49] X. Gao, C. Xu, H. Yin, X. Wang, Q. Song, P. Chen, Preparation of graphene by electrical explosion of graphite sticks, Nanoscale, 2017, to be published.

[50] T.W. Ebbesen, H. Hiura, Graphene in 3-dimensions: towards graphite origami, Advanced Materials, 7 (1995) 582-586.

[51] S. Zhu, T. Li, Hydrogenation-assisted graphene origami and its application in programmable molecular mass uptake, storage, and release, ACS Nano 8 (2014) 2864-2872.

[52] C.H. Jin, J.Y. Wang, Q. Chen, L.M. Peng, In situ fabrication and graphitization of amorphous carbon nanowires and their electrical properties, J. Phys. Chem. B 110 (2006) 5423-5428.

[53] Z. Aslam, R. Nicholls, A. Koos, N. Nicolosi, N. Grobert, Current-induced restructuring and chemical modification of $\mathrm{N}$-doped multi-walled carbon nanotubes, Advanced Functional Materials 21 (2011) 3933-3937.

[54] Z. Aslam, R. Nicholls, A.A. Koos, V. Nicolosi, N. Grobert, Investigating the structural, electronic, and chemical evolution of B-doped multi-walled carbon nanotubes as a result of joule heating, J. Physical Chemistry C 115 (2011) 2501925022.

[55] A. Bajpai, Z. Aslam, S. Hampel, R. Klingeler, and N. Grobert, A carbon-nanotube based nano-furnace for in-situ restructuring of a magnetoelectric oxide, Carbon 114 (2017) 291-300.

[56] P.M.F.J. Costa, U.K. Gautam, Y. Bando, D. Golberg, The electrical delivery of a sublimable II-VI compound by vapor transport in carbon nanotubes, Carbon 49 (2011) 3747-3754

[57] P.M.F.J. Costa, U.K. Gautam, Y. Bando, D. Golberg, Direct imaging of Joule heating dynamics and temperature profiling inside a carbon nanotube interconnect, Nature Commun. 2 (2011) 421. 


$$
0^{5}
$$




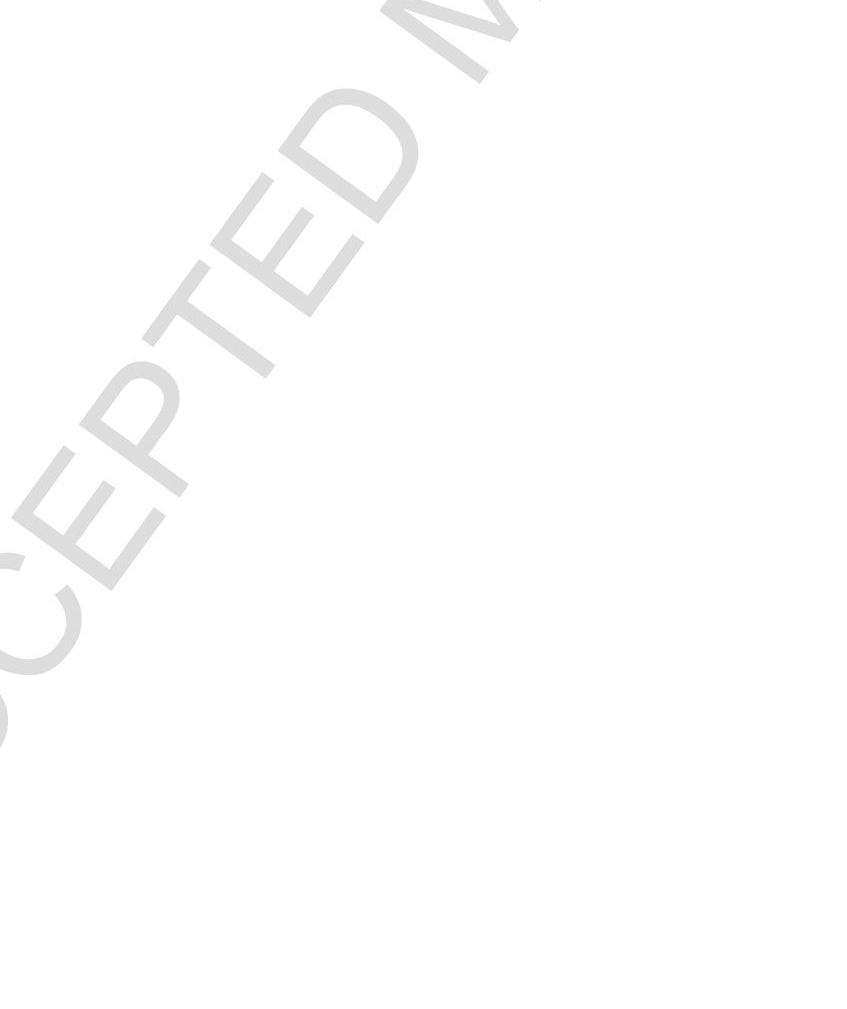





$$
5
$$





$$
5
$$




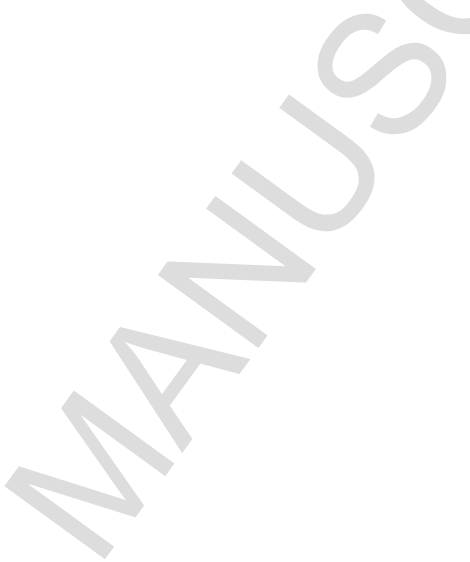




$$
5^{5}
$$




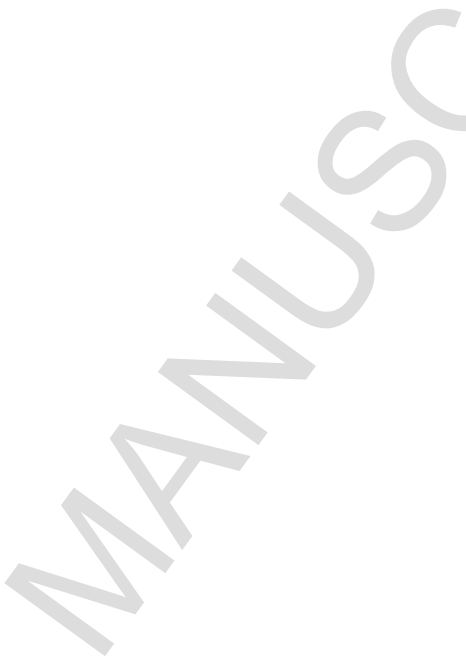





$$
5^{5}
$$






$$
5
$$





$$
0^{5}
$$

